\title{
Simulações Numéricas da Formação de Ilha de Calor na Região Metropolitana do Rio de Janeiro
}

Numericals Simulation of the Heat Island Formation in the Rio de Janeiro Metropolitan Region

Nilton Oliveira Moraes ${ }^{12}$; Edilson Marton ${ }^{1} \&$ Luiz Cláudio Gomes Pimentel ${ }^{1}$

${ }^{1}$ UFRJ, Departamento de Meteorologia, Instituto de Geociências - IGEO, Centro de Ciências Matemáticas e da Natureza - CCMN, Universidade Federal do Rio de Janeiro.e-mail:nilton@meteoro.ufrj.br,emarton@acd.ufrj.br,pimentel@acd.ufrj.br

${ }^{2}$ PEM/COPPE/UFRJ - Cidade Universitária - CT - Bloco G Ilha do Fundão - Rio de Janeiro - RJ

Recebido em: 23/10/2005 Aprovado em 30/10/2005

\section{Resumo}

As características diferenciadas de cobertura do solo da Região Metropolitana do Rio de Janeiro, onde encontramos regiões com um elevado percentual de áreas cobertas por asfalto e concreto, circundado por áreas rurais, determinam o aparecimento de um gradiente horizontal de temperatura conhecido como o fenômeno da ilha de calor urbana. Além dos aspectos locais referentes à ocupação do solo, diversos trabalhos científicos têm demonstrado a influência da circulação atmosférica de mesoescala e da escala sinótica no estudo desse fenômeno.

O principal objetivo do presente estudo consiste em observar e analisar, a partir de simulações com o modelo de mesoescala MM5, em conjunto com dados observacionais, a influência de sistemas sinóticos e características locais da RMRJ na formação e dissipação de ilha de calor urbana.

Palavras-chave: ilha de calor, efeitos sinóticos, modelo de mesoescala MM5.

\section{Abstract}

The differents characteristics of ground covering in the Rio de Janeiro Metropolitan Region, where regions with high percentage of asphalt and 
concrete are surrounded for rural areas, determine the appearance of a temperature horizontal gradient knowledge as urban heat island phenomenon. Besides, diverse scientific works have demonstrated the influence of the mesoescale atmospheric circulation and synoptic scale in the urban heat island formation and dissipation.

The main subjetive of study consists in analyze from simulated results, with the mesoescale model MM5, and observacionals data, the influence of synoptic systems and locals characteristics of the RMRJ in the formation and dissipation of urban heat island.

Keywords: Heat island, synoptic effects, MM5 Mesoscale Model.

\section{Introdução}

A Região Metropolitana do Rio de Janeiro (RMRJ) possui alta densidade populacional e elevado grau de industrialização, tendo como conseqüência uma grande concentração das fontes de emissão de poluentes. As peculiaridades topográficas e atmosféricas da região afetam diretamente a dispersão destes poluentes que, por sua vez, interferem nas mais diversas atividades humanas. Além disso, a característica de uso do solo da RMRJ apresenta um elevado percentual de áreas cobertas por asfalto e concreto, as quais são superfícies capazes de converter e armazenar a radiação solar incidente em maior grau que as áreas rurais. O conjunto dos fatores acima é capaz de determinar 0 aparecimento de um gradiente horizontal de temperatura conhecido como o fenômeno da ilha de calor urbana (Stull,1993). Estudos científicos apresentam a influência da ilha de calor urbana agindo sobre o campo de vento na camada limite atmosférica, alterando o comportamento da dispersão de poluentes na atmosfera. Em contrapartida, a presença de poluentes no ar modificam o balanço radiativo e o transporte de calor através da atmosfera. Um estudo realizado por Lombardo (1985) na Região Metropolitana de São Paulo sobre ilha de calor, constatou que situações sinóticas favoráveis à concentração de poluentes na atmosfera também contribuem para a intensificação do gradiente horizontal de temperatura em São Paulo. Situações como subsidência, inversão térmica, calmaria, umidade relativa baixa e névoa seca são condições favoráveis à formação dos fenômenos da ilha de calor e concentração de poluentes na atmosfera. Tudo isso, além de demonstrar a inter-relação entre o fenômeno da ilha de calor e da dispersão de poluentes atmosféricos, mostra que existe uma necessidade de se inserir a influência dos efeitos das circulações de mesoescala e da escala sinótica no estudo desses fenômenos. 
O principal objetivo do presente estudo consiste em observar e analisar, a partir de resultados simulados com o modelo de mesoescala MM5, a influência de sistemas sinóticos e características locais da RMRJ na formação e dissipação de ilha de calor urbana.

\section{Aspectos da Ilha de Calor Urbana}

A caracterização da ilha de calor urbana através do surgimento de gradientes horizontais de temperatura (Stull, 1993) pode, de maneira geral, ser controlada por diversos fatores. Dentre os principais fatores, destaca-se a caracterização da cidade (corpos d'água, natureza do solo, vegetação, uso do solo, arquitetura, os materiais de construção e fontes antropogênicas), localização geográfica, topografia, climatologia urbana, sazonalidade e condições sinóticas do tempo (Oke, 1982). Estudos de cunho observacional têm grande importância na determinação de parâmetros de superfície e atmosféricos que são relevantes na formação e manutenção dos gradientes térmicos horizontais. Oke (1982) relata uma série de artigos envolvendo a ilha de calor urbana, destacando o balanço de energia em áreas rurais, urbanas e suburbanas. A influência das condições atmosféricas na escala de tempo sazonal foi abordada por Acckerman (1985). Seus resultados, extraídos de uma série temporal de 20 anos de dados, indicam que fatores sazonais tendem a modular o ciclo diurno na ilha de calor urbana. Katsoulis \& Theoharatos (1985) analisaram registros de temperatura do ar no período de 22 anos (1961-1982), cobrindo a área de maior densidade populacional da cidade de Atenas. Seus resultados mostram um aumento da temperatura mínima média no período de estudo relacionado com o crescimento urbano. O trabalho de Balling \& Cerveny (1986) associa a média mensal da intensidade dos ventos com o desenvolvimento da ilha de calor urbana em Phoenix, EUA. Os autores concluíram que as mudanças observadas no campo de vento em Phoenix estão relacionadas diretamente com a da ilha de calor urbana, devido ao aumento do gradiente de temperatura entre a área metropolitana e os seus arredores. O estudo de Maitelli et al. (2004) investiga características espaciais e temporais da ilha de calor em Cuiabá/MT, utilizando estações móveis e de ponto fixo. Os resultados revelam que a ilha de calor localiza-se no distrito comercial, com elevação da temperatura de até $1.5^{\circ} \mathrm{C}$ nos dias chuvosos e de até $3.0^{\circ} \mathrm{C}$ em dias sem chuva. A técnica do sensoriamento remoto também é bastante utilizada no estudo de ilhas de calor. Souza \& Massambani (2004) utilizaram o índice de vegetação (NDVI) e 
Simulações Numéricas da Formação de Ilha de Calor na Região Metropolitana do Rio de Janeiro Nilton Oliveira Moraes; Edilson Marton \& Luiz Cláudio Gomes Pimentel

temperatura da superfície na identificação de ilha de calor na região metropolitana de São Paulo.

Do ponto de vista da modelagem numérica, simulações com os modelos MM5 (Montavez et al., 2003 ; Kinouchi \& J. Yoshitani, 2001 ; Sailor et al., 2003) e RAMS - Regional Atmospheric Model - (Freitas \& Silva Dias, 2000, 2003), dentre outros modelos, têm sido bastante utilizadas para o desenvolvimento de estudos sobre a estrutura interna e os mecanismos de formação de ilha de calor. Estudos desse tipo com modelos atmosféricos exigem alta resolução espacial. Portanto, geralmente utiliza-se domínios com grades aninhadas como em Sailor et al. (2003), em que foram definidas três grades com espaçamentos entre pontos de 18, 6 e $2 \mathrm{~km}$, respectivamente.

\section{Metodologia}

O estudo de ilha de calor neste trabalho é conduzido através de simulações numéricas da atmosfera, utilizando o modelo de mesoescala MM5. O modelo numérico atmosférico PSU/NCAR Mesoscale Modeling System MM5 Versão 3 (Dudhia et al. 2000) é um modelo não-hidrostático, de área limitada, que utiliza coordenada vertical sigma-p para o contorno do terreno. Possui uma estrutura modular composta por diversos programas auxiliares, organizados em módulos independentes, responsáveis pela geração de informações necessárias para a simulação. O módulo Terrain é o primeiro módulo do MM5, sendo responsável pela configuração do domínio do modelo, com opção de uso de grades aninhadas. Ele realiza uma interpolação horizontal sobre os dados de topografia e vegetação baseada em latitude-longitude sobre os domínios de mesoescala escolhidos. Efetua a leitura de arquivos contendo dados de elevação de terreno, máscara terra-água, tipos de solo, fração de vegetação e temperatura de sub-solo. A partir destes dados são gerados arquivos de entrada para os módulos seguintes. O módulo Regrid faz a leitura dos dados meteorológicos (análises e prognósticos) arquivados em uma grade regular em níveis de pressão. Realiza uma interpolação bi-dimensional nestes dados, adaptando-os às grades geradas pelo Terrain. A partir daí, são gerados arquivos que serão utilizados pelos módulos posteriores como análises para serem interpoladas diretamente para geração das condições de fronteira que serão utilizadas posteriormente nos módulos Interpf e MM5. No módulo Interpf são gerados os arquivos contendo as condições iniciais e de contorno que o modelo MM5 utilizará para realizar a simulação final. Estes arquivos são gerados a partir dos dados do 
módulo Regrid. No módulo chamado MM5 são resolvidas às equações diagnósticas e prognósticas do modelo matemático, e os resultados são passados para cada uma das parametrizações físicas pertinentes e processados por elas, a cada passo de tempo. Estas equações são as de transportes de quantidade de movimento, calor e umidade. O modelo traz diversas opções de parametrizações físicas para processos de superfície e camada limite, precipitação, microfísica de nuvens e radiação. A parametrização de cúmulos utilizada é a de Grell (Grell, 1993, 1995) e a de microfísica de nuvens é o esquema chamado Simple Ice, desenvolvido por Dudhia (1989), em que os campos de água de chuva e nuvem são prognosticados explicitamente com processos microfísicos.

Para a camada limite atmosférica utilizou-se o esquema MRF, implementado por Troen e Mahrt (1986). O modelo de solo é o desenvolvido por Dudhia (1996), que utiliza cinco camadas com espessuras de 1, 2, 4, 8 e 16 $\mathrm{cm}$ e um substrato com espessura de $31 \mathrm{~cm}$ e que é mantido a temperatura constante. O esquema radiação utlizado é o desenvolvido por Dudhia (1989).

Nas simulações desenvolvidas utilizaram-se dois domínios centralizados na posição correspondente ao Aeroporto Internacional do Galeão, sendo o segundo domínio aninhado ao primeiro de menor resolução espacial. O domínio maior possui resolução espacial na direção horizontal de $9 \mathrm{~km}$ ocupando uma extensão de 468 x 360 km, abrangendo todo o Estado do Rio de Janeiro, enquanto o segundo domínio, aninhado ao primeiro, possui resolução espacial de $3 \mathrm{~km}$ ocupando uma extensão de 93 x $93 \mathrm{~km}$. As resoluções topográficas utilizadas foram, respectivamente, $9 \mathrm{~km}$ e $1 \mathrm{~km}$. Foram utilizados dados de topografia e elevação da USGS (United State Geological Survey) para iniciar o primeiro módulo do modelo (Terrain). Foram utilizados 55 níveis verticais nos dois domínios, sendo 26 níveis até $850 \mathrm{hPa}$, para melhor resolver fenômenos existentes na camada limite atmosférica. O modelo foi inicializado com dados meteorológicos do conjunto de reanálies do NCEP (National Centers for Environmental Prediction) com resolução espacial na direção horizontal de $2.5^{\circ}$ lat/lon e a cada 6 horas.

\section{Resultados}

A seguir, serão apresentados alguns aspectos atmosféricos do mês de setembro de 2002 e uma análise mais detalhada de dois períodos com características sinóticas distintas. A discussão dos resultados será focada na avaliação da influência de sistemas sinóticos e dos efeitos atmosféricos locais 
sobre a formação de ilha de calor na RMRJ, interpretando-se simulações do campo de temperatura realizadas com o modelo MM5.

\subsection{Análise Sinótica do Mês de Setembro de 2002}

A análise sinótica do mês de setembro de 2002 é feita a partir da interpretação de cartas de pressão em superfície nos horários de 00 e 12Z, confeccionadas pela DHN - Marinha do Brasil, e de imagens do satélite GOES 8 no canal espectral infravermelho. Adicionalmente, são utilizados dados de reanálise do NCEP para um ponto localizado no oceano na latitude $25^{\circ} \mathrm{S}$ e longitude $45^{\circ} \mathrm{W}$, próximo ao litoral do Rio de Janeiro, permitindo acompanhar a variação temporal da pressão e do vento para todo o mês.

De acordo com as informações do CPTEC (Centro de Previsão do Tempo e Estudos Climáticos), oito sistemas frontais atuaram no litoral do Brasil em setembro, sendo que o primeiro deles teve origem no mês anterior. Na cidade do Rio de Janeiro houve a passagem de quatro desses sistemas frontais nos dias 1, 7, 14 e 21.

Na figura 1 apresentamos a evolução temporal da pressão e do vento em superfície para o mês de setembro de 2002 a partir dos dados de reanálise do NCEP no ponto de $25^{\circ} \mathrm{S}$ e $45^{\circ} \mathrm{W}$. Durante o mês é possível identificar quatro períodos de alta pressão na região: entre os dias 3-6, 8-12, 15-19 e 2230 (Figura 1.a). Nesses períodos os ventos são predominantemente do quadrante nordeste (Figura 1.b). Ocorreram também três períodos de baixa pressão: entre os dias 6-7, 12-15 e 20-22. Esses períodos de baixa pressão, podem ser relacionados com as passagens das frentes frias nos dias 7, 14 e 21, através da mudança de direção do vento de quadrante norte para o quadrante sudoeste/ sul, como apresentado na Figura 1.b.

A partir da análise de todo o mês de setembro, foram selecionados dois períodos com situação sinótica distinta para o detalhamento da análise sinótica e estudo da influência dos sistemas sinóticos sobre a formação e dissipação da ilha de calor na RMRJ.

O primeiro período (P1), entre os dias 6 a 8, é caracterizado pela passagem de um sistema frontal e o segundo período (P2), entre os dias 17 a 19, pela atuação marcante do Anticiclone Subtropical do Atlântico (ASA). Uma análise sinótica mais detalhada sobre cada período será apresentada a seguir. 
Simulações Numéricas da Formação de Ilha de Calor na Região Metropolitana do Rio de Janeiro Nilton Oliveira Moraes; Edilson Marton \& Luiz Cláudio Gomes Pimentel
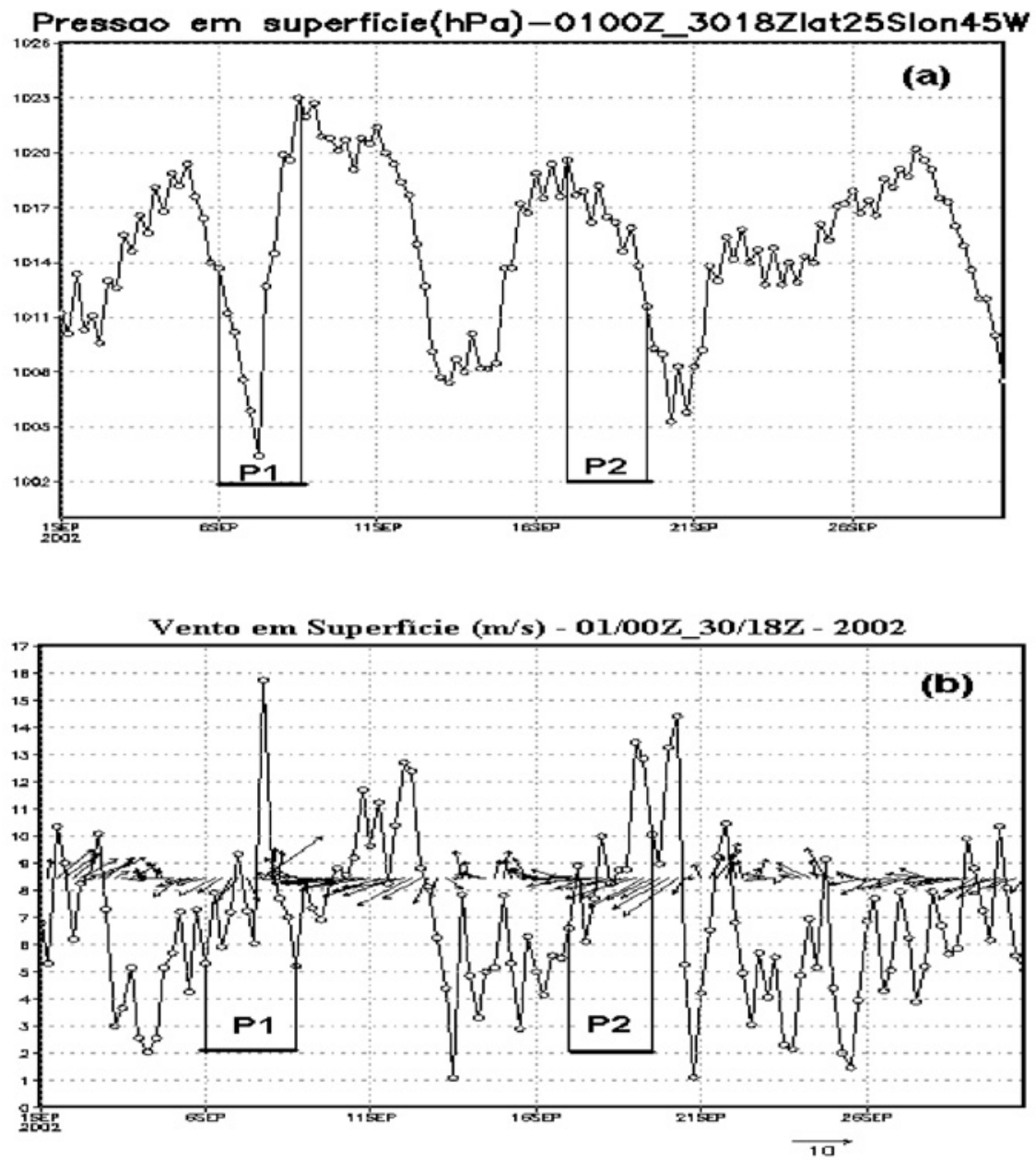

Figura 1 Evolução temporal durante o mês de setembro 2002 em $25^{\circ} \mathrm{S}$ e $45^{\circ} \mathrm{W}$ para (a) pressão em superfície (hPa) e (b) vento (m/s) em $1000 \mathrm{hPa}$. Em (b) as setas indicam a direção do vento e a curva contínua a respectiva intensidade. Os períodos P1 (dias 6 a 8) e P2 (dias 17 a 19) são destacados na parte inferior das figuras. Os dados são da reanálise do NCEP.

\subsubsection{Análise Sinótica do Período P1: 06 a 08 de setembro}

O período entre os dias 6 e 8 é caracterizado pela passagem de um sistema frontal no RJ. No dia 6 existe um cavado em superfície no litoral do Rio Grande do Sul que se intensifica com o tempo caracterizando, posteriormente, o sistema frontal que atua no sul do litoral de São Paulo no dia 7 às 00Z (Figura 2a). 


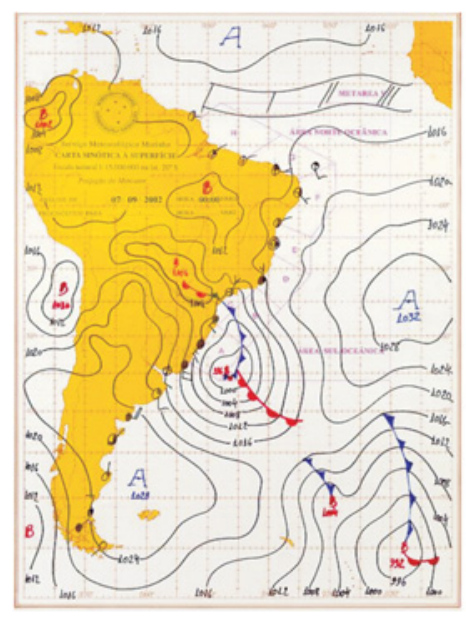

(a)

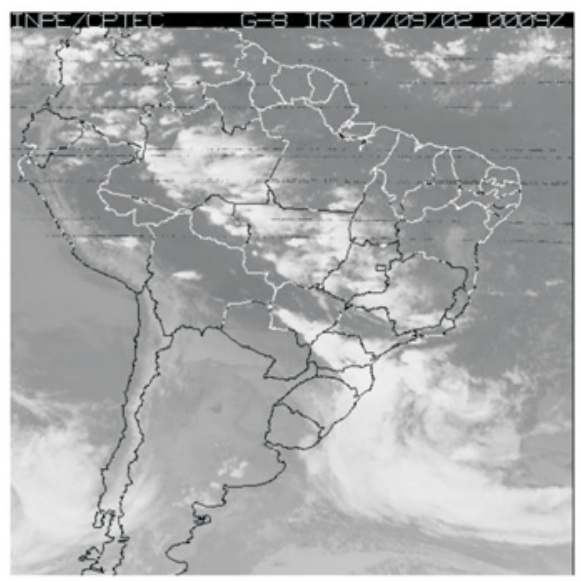

(b)

Figura 2 Dia 07/09 (a) Carta Sinótica as 00Z ; (b) Imagem de Satélite as 00Z;

O sistema frontal continua se deslocando ao longo do litoral, atinge o Espírito Santo no dia 8 e posiciona-se no sul da Bahia às $12 \mathrm{Z}$ do mesmo dia (Figuras 3a e 4a). As séries temporais de pressão e vento apresentadas na figura 1 ajudam a identificar o momento da passagem deste sistema sinótico no RJ. Note-se a queda acentuada da pressão e mudança do vento do quadrante nordeste para sudeste entre 12 e 18Z. No dia 8 às $00 \mathrm{Z}$ é notável a elevação acentuada da pressão e o registro de ventos de sul/ sudeste. Portanto, uma situação pré-frontal fica bem caracterizada na primeira metade do dia 7, enquanto que o dia 8 é tomado por uma situação pós-frontal. A nebulosidade associada ao sistema frontal pode ser acompanhada através das figuras ( $2 b, 3 b$ e $4 b$ ). O sul do Brasil encontrase parcialmente coberto por essas nuvens no dia 7 , enquanto que pouca nebulosidade atinge o RJ às $00 \mathrm{Z}$.

No dia 8, a nebulosidade apresenta-se melhor organizada em uma faixa, principalmente às 00Z, estendendo-se desde o Brasil Central e passando pela maior parte da Região Sudeste. No horário das $12 Z$ do dia 8 o RJ encontrase sob o domínio da massa polar que avança na retaguarda da frente fria. Pela imagem de satélite vemos que existe uma faixa de nebulosidade baixa sobre o RJ e que se estende pelo litoral até a Região Sul, configurando uma situação típica de circulação pós-frontal. 
Simulações Numéricas da Formação de Ilha de Calor na Região Metropolitana do Rio de Janeiro Nilton Oliveira Moraes; Edilson Marton \& Luiz Cláudio Gomes Pimentel

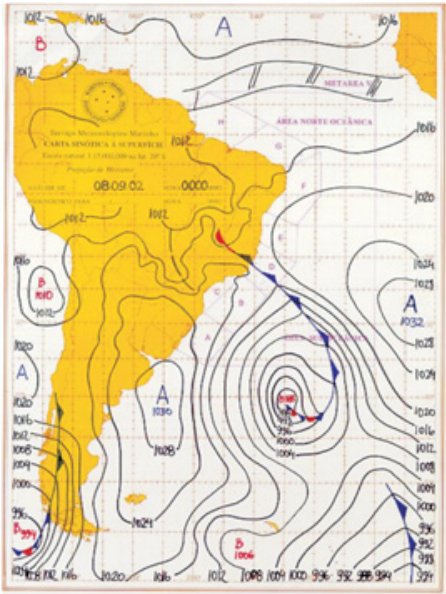

(a)

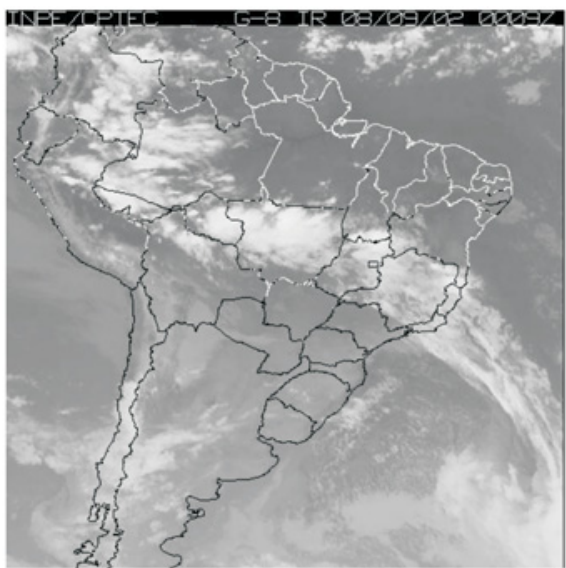

(b)

Figura 3 Dia 08/09 (a) Carta Sinótica as 00Z ; (b) Imagem de Satélite as 00Z.

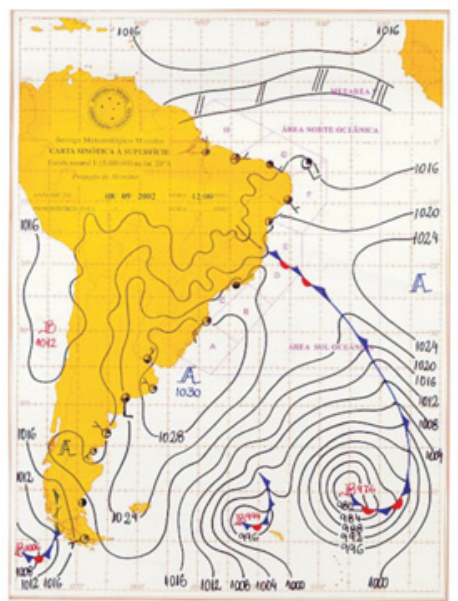

(a)

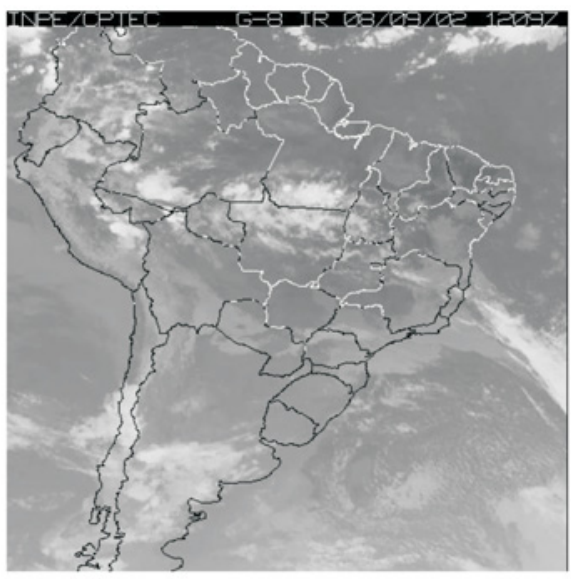

(b)

Figura 4 Dia 08/09 (a) Carta Sinótica as 12Z ; (b) Imagem de Satélite as 12Z 
Simulações Numéricas da Formação de Ilha de Calor na Região Metropolitana do Rio de Janeiro Nilton Oliveira Moraes; Edilson Marton \& Luiz Cláudio Gomes Pimentel

\subsubsection{Análise Sinótica do Período P2: 17 a 19 de Setembro}

No período entre os dias 17/09 e 19/09 o RJ é caracterizado pela influência marcante do anticiclone subtropical do Atlântico (ASA). As Figuras 5a, 6a e 7a ilustram o comportamento da pressão em superfície às $00 \mathrm{Z}$ no período. Note-se que o centro do ASA sofre um ligeiro deslocamento na direção nordeste, apresentando valores de pressão por volta de $1040 \mathrm{hPa}$ nos três dias. Este padrão semi-estacionário do ASA confere uma característica peculiar neste período, pois impede que perturbações atmosféricas de escala sinótica consigam penetrar no continente. Um exemplo claro desta situação pode ser observado nos dias 18 e 19, em que um centro de baixa pressão no extremo sul da América do Sul, associado a um sistema frontal, se desloca para leste sem atingir o continente. Outra conseqüência deste quadro sinótico é que o RJ permanece influenciado pelo setor noroeste do ASA. Esse aspecto é acompanhado, portanto, de ventos persistentes de nordeste na região, favorecendo a advecção de ar quente proveniente de latitudes mais ao norte, como pode ser observado nos registros de vento da própria carta sinótica e pela Figura 2b. O acompanhamento das imagens de satélite mostra que a nebulosidade mais intensa e organizada ocorre na Região Sul e no Atlântico Sul, evidenciando pouca nebulosidade no RJ no período (Figuras 5b, 6b e 7b).

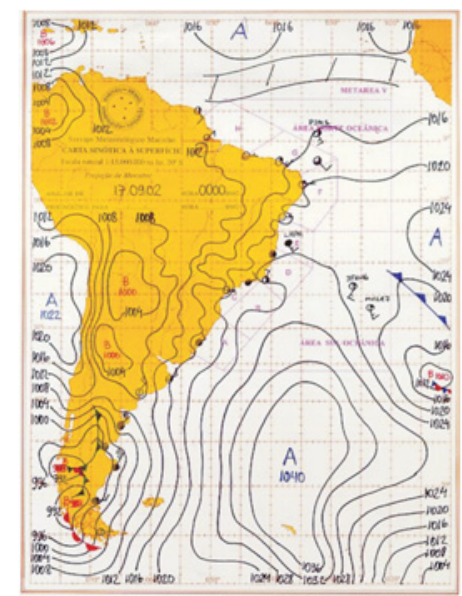

(a)

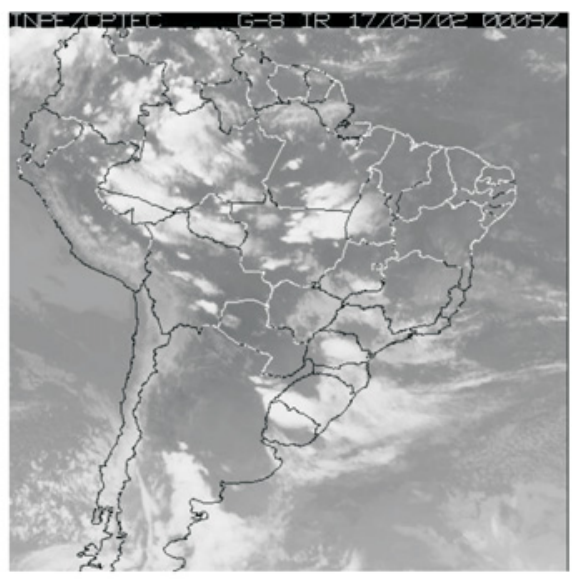

(b)

Figura 5 Dia 17/09 (a)Carta Sinótica as 00Z ; (b) Imagem de Satélite as 00Z 
Simulações Numéricas da Formação de Ilha de Calor na Região Metropolitana do Rio de Janeiro Nilton Oliveira Moraes; Edilson Marton \& Luiz Cláudio Gomes Pimentel

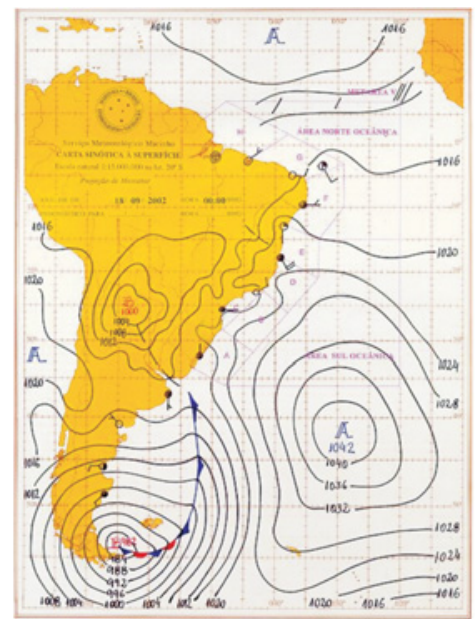

(a)

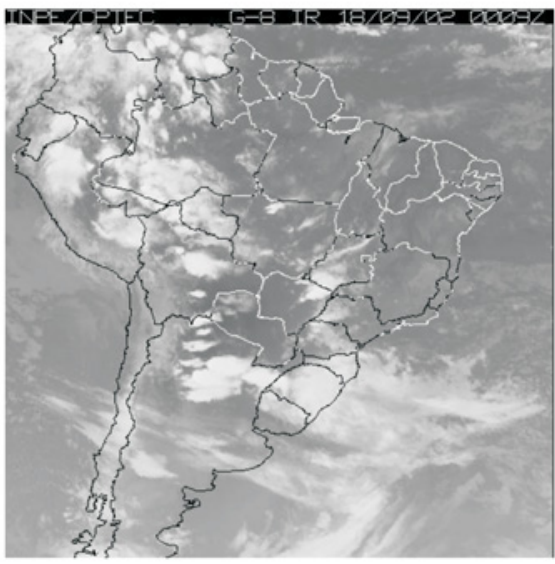

(b)

Figura 6 Dia 18/09 (a)Carta Sinótica as 00Z ; (b) Imagem de Satélite as 00Z.

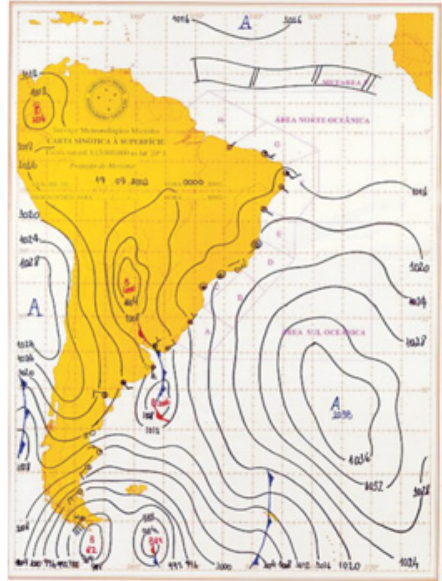

(a)

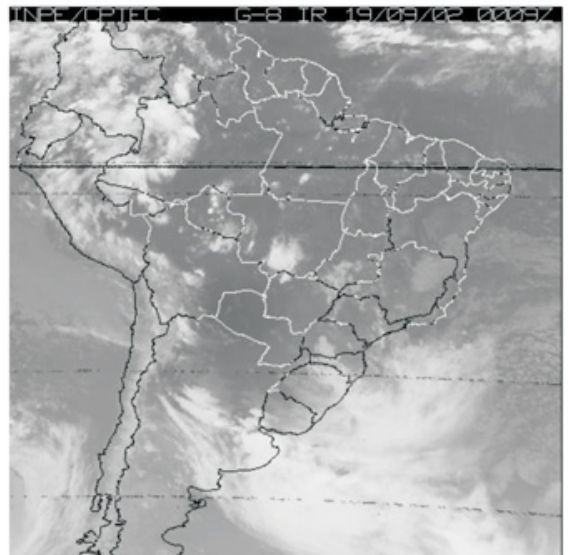

(b)

Figura7 Dia 19/09 (a)Carta Sinótica as 00Z ; (b) Imagem de Satélite as 00Z 


\subsection{Estudo da Formação de Ilha de Calor Urbana sob a Influência de Diferentes Situações Sinóticas.}

A seguir, serão apresentados resultados de simulações numéricas com o modelo MM5 para discutir a formação de ilha de calor, evidenciando o aspecto urbano, juntamente com a influência da circulação atmosférica de escala sinótica. O enfoque dado nesta análise será na variação temporal e espacial da temperatura próximo à superfície, uma vez que esta variável está fortemente relacionada ao fenômeno de ilha de calor.

A figura 8 ilustra a variação temporal da temperatura média espacial próximo à superfície em uma área correspondente a RMRJ, para todo o mês de setembro. O ciclo diurno da temperatura é bastante evidente, apresentando valores máximos e mínimos por volta das $18 \mathrm{Z}$ e 09Z, respectivamente, caracterizando uma oscilação com períodos definidos na curva de temperatura. Note-se que o período P1 está inserido em um período maior de elevação de temperatura com início a partir do dia 3, atingindo seu valor máximo no horário de $12 Z$ do dia 07 . A partir daí ocorre uma queda gradual da temperatura, sendo o valor mínimo às $12 \mathrm{Z}$ do dia 08 . Esse comportamento, obtido a partir da simulação, está concordante com a situação sinótica anteriormente descrita, onde notamos uma situação pré-frontal ocorrendo no dia 07 e pós-frontal no dia 08.

Por outro lado, durante todo o período P2 as temperaturas tiveram um comportamento muito similar de um dia para outro, variando com o ciclo diurno. As temperaturas atingiram os seus valores máximos no horário de $18 \mathrm{Z}$ dos dias 17, 18 e 19, sendo o dia 18 o de valor máximo no período. Os resultados da simulação apresentaram um comportamento físico coerente com a situação sinótica de influência do ASA no RJ. 


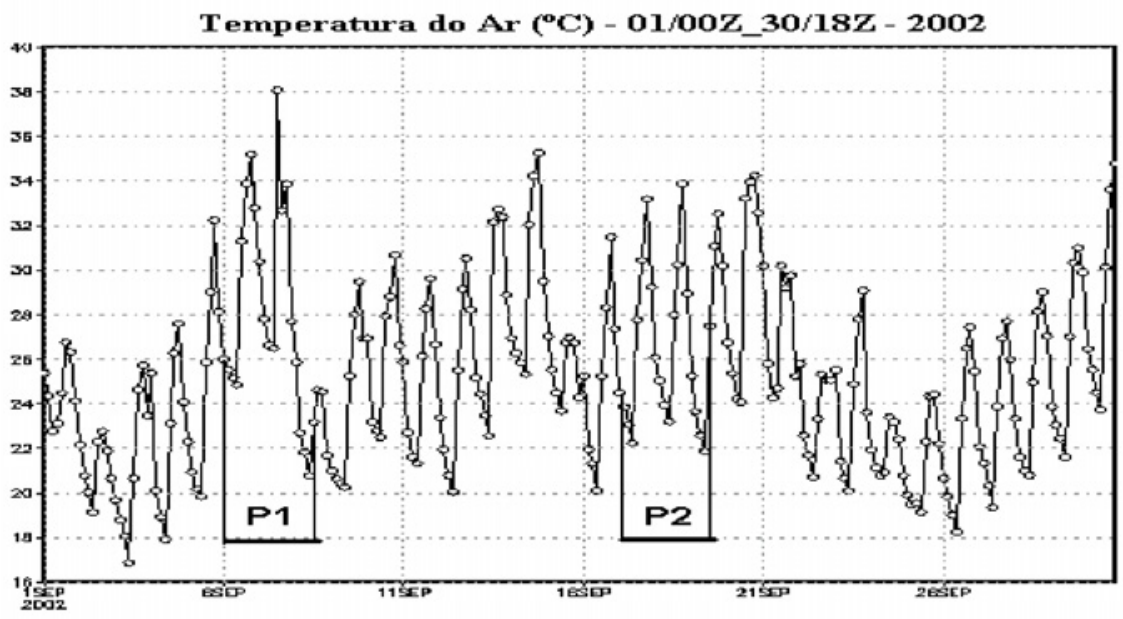

Figura 8 Evolução temporal da temperatura $\left({ }^{\circ} \mathrm{C}\right)$ no mês de Setembro 2002. Simulada pelo MM5

\subsubsection{Período P1: 06 a 08 de setembro}

A evolução temporal da distribuição horizontal da temperatura próximo a superfície é mostrada nas figuras 9, 10 e 11 para os dias 6, 7 e 8 nos horários de 12, 15, 18 e 21Z, respectivamente. Uma análise do dia 6 evidencia o aumento da temperatura no decorrer do dia, apresentando altos valores em uma região maior que a da RMRJ. Entretanto, o máximo de temperatura ocorre na própria RMRJ. Percebe-se que durante o ciclo diurno da temperatura o aquecimento ocorre mais intensamente no norte da RMRJ (12Z). Durante o processo de resfriamento, ao entardecer, o norte da RMRJ novamente se destaca por ainda apresentar máximos de temperatura (21Z). Uma comparação no horário das $12 \mathrm{Z}$ entre os dias 6 e 7 revela temperaturas mais elevadas no dia 7 (acima de $40^{\circ} \mathrm{C}$ ) na RMRJ. A análise sinótica feita na seção 4.1, em conjunto com os dados de reanálise, indica uma situação de pré-frontal no dia 7, o que demonstra a coerência dos resultados da simulação com relação à intensificação da temperatura.

No dia 07, ocorre a passagem de um sistema frontal, provocando alterações significativas na direção do vento por volta das $18 \mathrm{Z}$, conforme discutido na seção 4.1 . Neste dia, temperaturas acima de $40^{\circ} \mathrm{C}$ são simuladas pelo modelo às $12 Z$ e 18Z, com ligeira queda no horário intermediário de $15 Z$. Em princípio, o comportamento esperado era da ocorrência de máxima 
temperatura somente às $18 \mathrm{Z}$. No entanto, esse comportamento variável da evolução da temperatura pode ser decorrente de diversos fatores tais como: nebulosidade e mudança no comportamento da circulação devido à proximidade da entrada da frente.

No dia 08 as temperaturas diminuem sensivelmente, como pode ser visto na figura 11, devido à passagem da frente fria no dia anterior. Mesmo sob influência da massa de ar fria que acompanha o sistema frontal no dia 8, é possível identificar que a temperatura na RMRJ é ligeiramente superior do que suas vizinhanças caracterizando, portanto, um ciclo diurno de temperatura com pequena amplitude.

Um aspecto que deve ser ressaltado neste período P1 é que o norte da RMRJ apresenta valores de temperatura mais elevados do que suas vizinhanças. Isto pode ser notado claramente nos dias 6 e 7 no decorrer do ciclo diurno, tanto no horário em que se dá início ao aquecimento próximo à superfície (12Z) como também no momento em que a superfície se resfria por perda radiativa no final da tarde e início da noite (21Z). É possível que as circulações locais de brisa marítima e terrestre sejam responsáveis pela atenuação dos valores de temperatura na linha da costa, através de processos advectivos. Esta influência local justificaria, portanto, a manutenção dos máximos de temperatura mais para dentro do continente, ou seja, no norte da RMRJ.

No dia 8 essa característica também pode ser notada, porém com menor intensidade devido a influência da massa de ar frio associado ao sistema frontal. Esse comportamento de máximas temperaturas no norte da RMRJ sugere a influência da urbanização na distribuição horizontal da temperatura, indicando a formação da ilha de calor.

\subsubsection{Período P2: 17 a 19 de setembro}

A distribuição espacial da temperatura próximo à superfície para o período P2 é apresentada nas figuras 12,13,14, respectivamente para os dias 17, 18 e 19 e nos horários de 12, 15, 18 e 21Z. Note-se que os máximos de temperatura sempre ocorrem às $18 Z$, como já discutido anteriormente na análise da série temporal apresentada na figura 8. É possível notar, também, que estes valores máximos permanecem concentrados na RMRJ, especialmente na sua parte norte, de forma semelhante ao período P1. A análise sinótica evidencia a influência do ASA neste período P2 e a manutenção de ventos de nordeste no RJ. Portanto, advecção positiva de temperatura é mantida por todo o período 
como conseqüência direta deste padrão de vento. A regularidade da intensidade dos máximos de temperatura é coerente com esse quadro sinótico e pode ser uma característica típica da influência do ASA na RMRJ. Uma comparação entre os períodos P1 e P2 revela que os máximos de temperatura são mais intensos em P1. Apesar de ambos os períodos sofrerem aquecimento por processos advectivos, a advecção positiva de temperatura em P1 indica ser mais intensa por incluir um episódio de pré-frontal.

\section{Conclusões}

Este trabalho discute a formação de ilha de calor urbana na RMRJ. A abordagem deste tema fez uso de simulações numéricas da atmosfera com o modelo MM5, sob diferentes situações sinóticas. Inicialmente, discutiu-se o estado da atmosfera para dois períodos no mês de setembro de 2002. O primeiro período (P1) é referente a passagem de um sistema frontal no RJ e o segundo (P2) é caracterizado pela atuação persistente do ASA. A série temporal da temperatura próximo à superfície em uma região representativa da RMRJ evidencia um ciclo diurno bem marcante com elevação dos máximos diários no decorrer de P1 e queda brusca da temperatura logo após a passagem do sistema frontal. No período P2, os máximos diários ficam praticamente estáveis, sugerindo uma característica típica de atuação do ASA com a permanência de ventos de nordeste na região. Na distribuição espacial da temperatura próximo à superfície, pode-se verificar que os valores mais elevados desta variável são encontrados em uma região maior do que a RMRJ nos dois períodos estudados. Entretanto, é possível notar que o máximo de temperatura coincide com a ocupação urbana da RMRJ, sobretudo em sua parte norte. Este aspecto indica a formação de ilha de calor urbana na região. Fazendo uma comparação entre os dois períodos analisados, verifica-se que em P1 os máximos de temperatura são mais intensos. Ventos de nordeste na região nos dois períodos certamente contribuíram para esses máximos, em decorrência da advecção positiva de temperatura. No entanto, o período P1 contempla um estado atmosférico préfrontal e este fato intensifica a advecção de temperatura justificando, ao menos em parte, as temperaturas mais elevadas em P1 quando comparadas com o período P2. O presente trabalho indica, portanto, que simulações numéricas da atmosfera interpretadas em conjunto com suas respectivas condições sinóticas, oferecem uma grande capacidade de prognóstico de ilha de calor urbana. 
Simulações Numéricas da Formação de Ilha de Calor na Região Metropolitana do Rio de Janeiro Nilton Oliveira Moraes; Edilson Marton \& Luiz Cláudio Gomes Pimentel
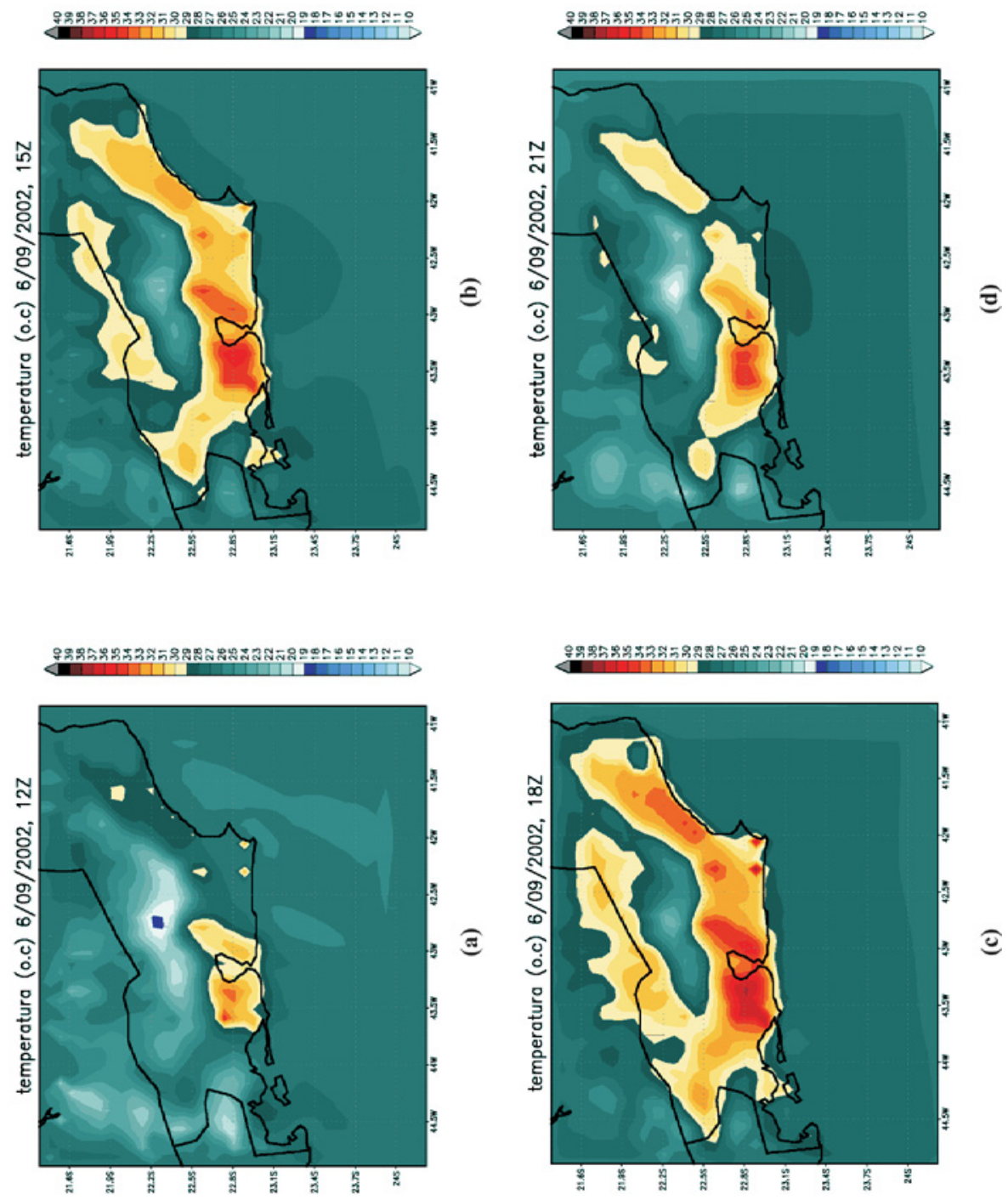

Figura 9 Campo de temperatura simulado pelo MM5 referente ao dia 06/09/2002 (a) 12 Z; (b) $15 \mathrm{Z}$; (c) $18 \mathrm{Z}$; (d) $21 \mathrm{Z}$. 
Simulações Numéricas da Formação de Ilha de Calor na Região Metropolitana do Rio de Janeiro Nilton Oliveira Moraes; Edilson Marton \& Luiz Cláudio Gomes Pimentel
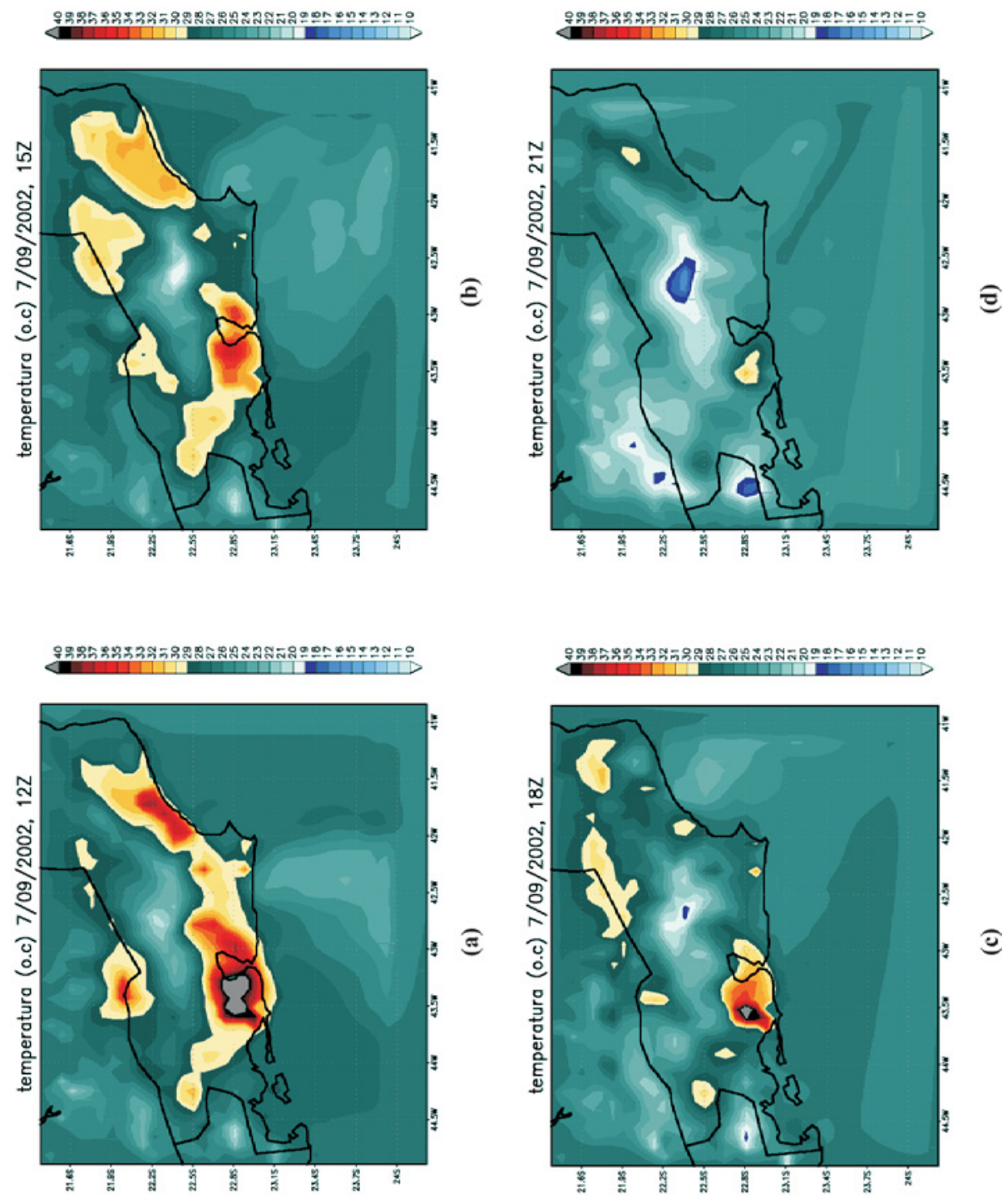

Figura 10 Campo de temperatura simulado pelo MM5 referente ao dia 07/09/2002 (a) 12 Z; (b) $15 \mathrm{Z}$; (c) $18 \mathrm{Z}$; (d) $21 \mathrm{Z}$. 
Simulações Numéricas da Formação de Ilha de Calor na Região Metropolitana do Rio de Janeiro Nilton Oliveira Moraes; Edilson Marton \& Luiz Cláudio Gomes Pimentel
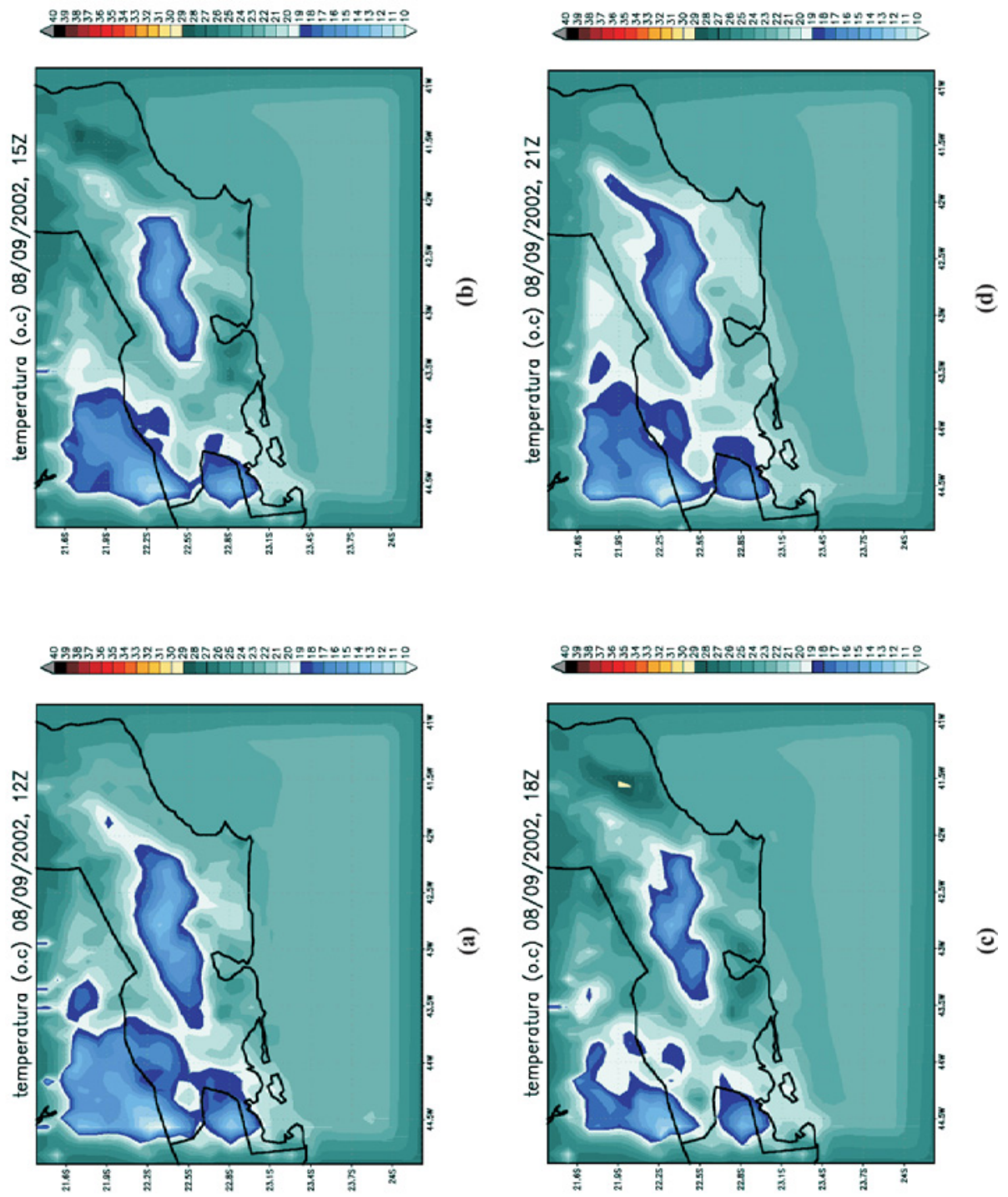

Figura 11 Campo de temperatura simulado pelo MM5 referente ao dia 08/09/2002 (a) 12Z; (b) 15Z; (c) 18Z; (d) $21 \mathrm{Z}$ 
Simulações Numéricas da Formação de Ilha de Calor na Região Metropolitana do Rio de Janeiro Nilton Oliveira Moraes; Edilson Marton \& Luiz Cláudio Gomes Pimentel
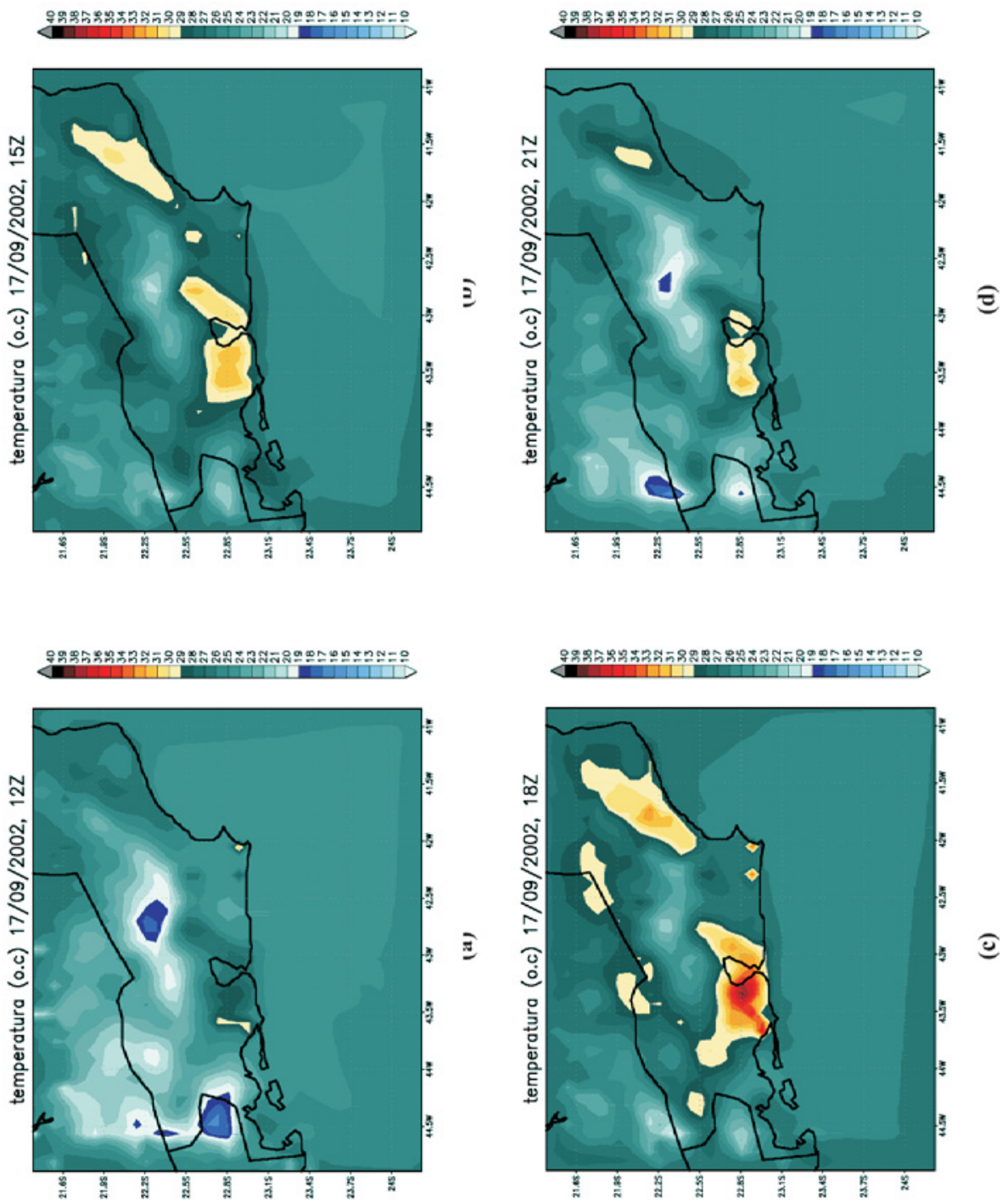

Figura 12 Campo de temperatura simulado pelo MM5 referente ao dia 17/09/2002 (a) 12Z; (b) $15 Z$; (c) $18 Z$; (d) $21 \mathrm{Z}$ 
Simulações Numéricas da Formação de Ilha de Calor na Região Metropolitana do Rio de Janeiro Nilton Oliveira Moraes; Edilson Marton \& Luiz Cláudio Gomes Pimentel
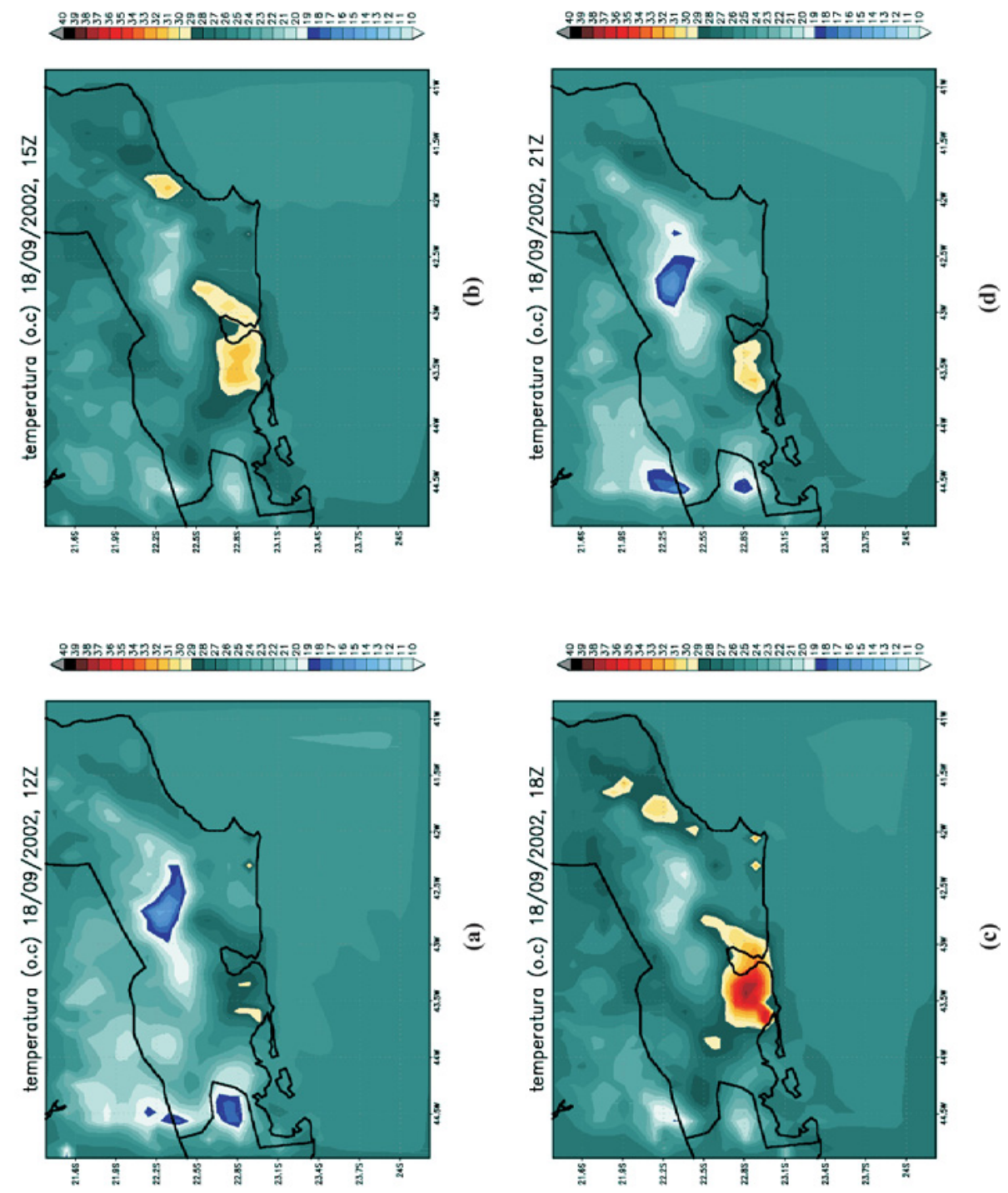

Figura 13 Campo de temperatura simulado pelo MM5 referente ao dia 18/09/2002 (a)12Z; (b) 15Z; (c) 18Z; (d)21Z 
Simulações Numéricas da Formação de Ilha de Calor na Região Metropolitana do Rio de Janeiro Nilton Oliveira Moraes; Edilson Marton \& Luiz Cláudio Gomes Pimentel
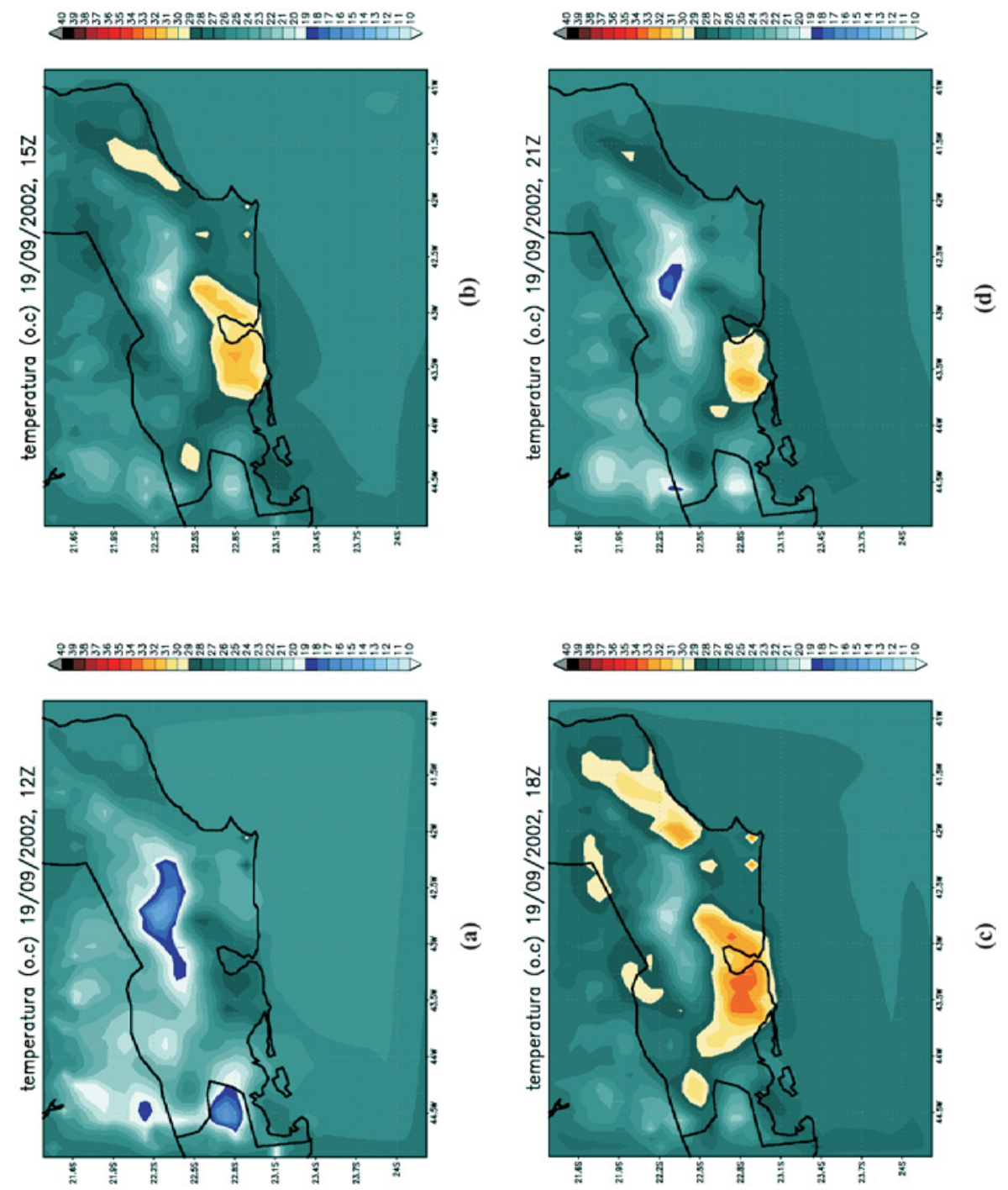

Figura 14 Campo de temperatura simulado pelo MM5 referente ao dia 19/09/2002 (a) $12 \mathrm{Z}$; (b) $15 \mathrm{Z}$; (c) $18 \mathrm{Z}$; (d) $21 \mathrm{Z}$ 
Simulações Numéricas da Formação de Ilha de Calor na Região Metropolitana do Rio de Janeiro Nilton Oliveira Moraes; Edilson Marton \& Luiz Cláudio Gomes Pimentel

\section{Referências}

Acckerman, B. 1985. Temporal March of the Chicago Heat Island. Journal of Applied Meteorology, 24(6): 547-554.

Balling, R. C. JR. \& Cerveny, R. S. 1986. Long-Term Associations between Wind Speeds and the Urban Heat Island of Phoenix, Arizona. Journal of Applied Meteorology, 26(6): 712-716.

Climanálise, 2002. Boletim de Monitoramento e Análises Climáticas. Cachoeria Paulista: INPE-CPTEC, São Paulo, 17.

Dudhia, J. 1989. Numerical study of convection observed during the winter monsoon experiment using a mesoscale two-dimensional model. $J$. Atmos. Sci., 46: 3077-3107.

Dudhia, J. 1996. A multi-layer soil temperature model for MM5. In: Preprint from the Sixth PSU/NCAR Mesoscale Model Users 'Workshop, 22-24 July 1996. Boulder, Colorado, p. 49-50

Dudhia, J.; Gill, D.; Guo, Y.; Manning, K.; Wang, W. \& Chiszar, J. 2001. Mesoscale Modeling System Tutorial Class Notes and User's Guide: MM5 Modeling System Version 3, PSU/NCAR. 255 p.

Freitas, E. F. \& Silva Dias, P .L. 2000. O Efeito da Ilha de Calor Sobre os Fluxos de Calor Através da Utilização do Modelo RAMS. In: CONGRESSO BRASILEIRO DE METEOROLOGIA,11, Rio de Janeiro, 2000. Anais, Rio de Janeiro, SBMET, p. 3566-3574.

Freitas, E. F. \& Silva Dias, P. L. 2003. A Importância das Fontes Antropogênicas de Calor na Modelagem Numérica dos Efeitos de Ilha de Calor Urbana. In: WORKSHOP BRASILEIRO DE MICROMETEOROLOGIA,3, Santa Maria, 2003. Revista Ciência \& Natura, Santa Maria, UFSM, p. 227-231.

Grell, G. 1993. Prognostic evaluation of assumptions used by cumulus parameterizations. Mon. Wea. Rev., 121: 764-787.

Grell, G. A.; Dudhia, J. \& Stauffer, D. R. 1995. A description of the fifthgeneration Penn State/ NCAR mesoscale model (MM5). NCAR Technical Note, NCAR/TN-398+STR, 117 p.

Katsoulis, B. D. \& Theoharatos, G. A. 1985. Indications of the Urban Heat Island in Athens, Greece, Journal of Applied Meteorology, 24(12): 1296-1302.

Kinouchi, T. \& Yoshitani, J. 2001. Simulation of the urban heat island in Tokyo with future possible increases of anthropogenic heat, vegetation cover and water surface. In: INTERNATIONAL SYMPOSIUM ON ENVIRONMENTAL HYDRAULICS,3, Geographical Survey Institute, Japão,2002.12. 
Simulações Numéricas da Formação de Ilha de Calor na Região Metropolitana do Rio de Janeiro Nilton Oliveira Moraes; Edilson Marton \& Luiz Cláudio Gomes Pimentel

Lombardo, M. A. 1985. Ilha de Calor nas Metrópoles: O Exemplo de São Paulo. São Paulo, Ed. HUCITEC, 244 p.

Montavez, J. P.; Gonzalez-Rouco, J. F. \& Valero F. 2003. A Study of The Three-Dimensional UHI by Using a Mesoscale Model. In: INTERNATIONAL CONFERENCE ON URBAN CLIMATE, 5, Lodz, Polônia,2003. Lodz, sessão pôster 2.

Maitelli, G. T.; Chileto, E. C.; Junior, N. L. .A. \& Chileto, R. 2004. Intensidade Da Ilha De Calor em Cuiabá/MT, na Estação Chuvosa. In: CONGRESSO BRASILEIRO DE METEOROLOGIA, 13, Fortaleza/ CE, 2004. Anais, Fortaleza, SBMET, p. 69-82.

Oke, T. R. 1982. The Energetic basis of the Urban Heat Island. Q. J. R. Meteorol. Soc., 108: 1-23.

Sailor, D. J.; Lu, L. \& Fan, H. 2003. Estimating Urban Anthropogenic Heating Profiles and Their Implications for Heat Island Development. INTERNATIONAL CONFERENCE ON URBAN CLIMATE,15, Lodz, Polônia,2003. Lodz, sessão oral 28.

Souza, A. L. F. \& Masssambani, O. 2004. Ilha de Calor Urbana na Região Metropolitana de São Paulo. In: CONGRESSO BRASILEIRO DE METEOROLOGIA, 13, Fortaleza/CE, 2004. Anais, Fortaleza, SBMET, p. 565- 584.

Stull, R. B. 1993. An Introduction to Boundary Layer Meteorology. Kluwer Academic Publishers, Dordrecht. 666 p.

Troen, I. \& Mahrt, L. 1986. A Simple Model of the Atmospheric Boundary Layer: Sensivity to Surface Evaporation, Boundary Layer. Meteorology, 37: 129-148. 